\title{
Thermal quarkonium mass shift from Euclidean correlators
}

\author{
Alexander M. Eller, ${ }^{1, *}$ Jacopo Ghiglieri, ${ }^{2, \dagger}$ and Guy D. Moore ${ }^{1, \hbar}$ \\ ${ }^{1}$ Institut für Kernphysik, Technische Universität Darmstadt Schlossgartenstraße 2, \\ D-64289 Darmstadt, Germany \\ ${ }^{2}$ Theoretical Physics Department, CERN, CH-1211 Genève 23, Switzerland
}

(Received 22 March 2019; published 31 May 2019)

\begin{abstract}
Brambilla, Escobedo, Soto, and Vairo have derived an effective description of quarkonium with two parameters; a momentum diffusion term and a real self-energy term. We point out that the real self-energy term can be expressed directly in terms of Euclidean electric-field correlators along a Polyakov line. This quantity can be directly studied on the lattice without the need for analytical continuation. We show that existing Minkowski-space calculations of this correlator correspond with the known NLO Euclidean value of the relevant electric-field two-point function.
\end{abstract}

DOI: 10.1103/PhysRevD.99.094042

\section{INTRODUCTION}

Quarkonium (bound heavy quark-antiquark states) are an intriguing probe of the quark-gluon plasma [1]. Originally proposed by Matsui and Satz [2], the suppression of quarkonia has remained an active topic of experimental [3-8] and theoretical [9-15] investigation ever since. The central idea is that a thermal medium tends to break up quarkonium bound states; one then investigates how strong this effect is expected to be theoretically and how much such states are suppressed experimentally. Recently, it has become clear that for charmonium at the highest energies, there are also important recombination effects from the many open charm quarks in the plasma [16-18].

Recently, Brambilla, Escobedo, Soto, and Vairo have used potential nonrelativistic QCD (pNRQCD) [19-21] at second order in the multipole expansion to rigorously derive, in Ref. [14], an open-quantum-system effective description for quarkonium evolution in a quark-gluon plasma for $m \gg 1 / a_{0} \gg T$, where $m$ is the heavy quark mass and $a_{0} \sim 1 /\left(m \alpha_{\mathrm{s}}\right)$ the Bohr radius. Their description depends on two (in principle, nonperturbative) parameters describing the interaction of the thermal medium with heavy quarks. One parameter is the well-known heavyquark momentum-diffusion coefficient [22],

\footnotetext{
*meller@theorie.ikp.physik.tu-darmstadt.de

jacopo.ghiglieri@cern.ch

guy.moore@physik.tu-darmstadt.de
}

Published by the American Physical Society under the terms of the Creative Commons Attribution 4.0 International license. Further distribution of this work must maintain attribution to the author(s) and the published article's title, journal citation, and DOI. Funded by SCOAP ${ }^{3}$.

$$
\kappa=\frac{g^{2}}{6 N_{c}} \operatorname{Re} \int_{-\infty}^{\infty} d s\left\langle\mathrm{~T} E^{a, i}(s, \mathbf{0}) E^{a, i}(0, \mathbf{0})\right\rangle,
$$

where $E^{a, i}$ is the color electric field, $s$ is the Minkowski time value, both $E$ fields are at the same space coordinate $\mathbf{0}$, $N_{c}=3$ is the number of colors, $\mathrm{T}$ is the time-ordering symbol, and a fundamental-representation forward-time Wilson line is implicitly included in the time-ordered correlator indicated. This quantity has been extensively investigated in the literature, in weak-coupling QCD [23-26], effective models [27], holographic dual theories $[22,28]$, and via analytical continuation from lattice data [29-33].

The second parameter is a real nondissipative plasma effect, which induces a mass shift in the heavy-quark bound states. At lowest order in pNRQCD, the shift is $\delta m=\frac{3}{2} a_{0}^{2} \gamma$ [10], where $\gamma$ is the following electric-field correlator:

$$
\gamma=\frac{g^{2}}{6 N_{\mathrm{c}}} \operatorname{Im} \int_{-\infty}^{\infty} d s\left\langle\mathrm{~T} E^{a, i}(s, \mathbf{0}) E^{a, i}(0, \mathbf{0})\right\rangle .
$$

This correlator has received less attention in the literature, and as we will show, it will actually be much easier to determine via lattice QCD methods than the coefficient $\kappa$. Therefore, in this paper, we focus on investigating the coefficient $\gamma$.

In the next section, we show how to analytically continue Eq. (2) to Euclidean time. This continuation leads to a time integral of a correlator of electric fields along a Polyakov loop. In the remainder of the paper, we check this derivation by showing that the known next-to-leading order (NLO) value of $\gamma$, derived by Minkowski space methods in Ref. [10], correctly corresponds to the appropriate integral moment of the known NLO Euclidean correlator from Ref. [34]. This is a nice check of our continuation. 
However, to us, the most interesting result is the possibility of a future nonperturbative determination of $\gamma$ on the lattice.

\section{ANALYTIC CONTINUATION OF ELECTRIC-FIELD CORRELATOR}

The analytic continuation of the electric-field correlator, Eq. (2), is not trivially doable. However, we do not consider this correlator directly but instead use the heavy-quark current-current correlator

$$
\int_{-\infty}^{\infty} d t e^{i \omega t} \int d^{3} \mathbf{x}\left\langle\left[\hat{\mathcal{J}}^{\mu}(t, \mathbf{x}), \hat{\mathcal{J}}^{\nu}(0, \mathbf{0})\right]\right\rangle,
$$

from which Eq. (2) originates. Here, $\hat{\mathcal{J}}^{\mu} \equiv \hat{\bar{\psi}} \gamma^{\mu} \hat{\psi}$ is the heavy-quark current, and $\hat{\psi}$ is the heavy-quark field operator. The definition of the heavy-quark momentumdiffusion coefficient $\kappa$ as the spectral function of Eq. (3) in the $M_{\text {kin }} \rightarrow \infty$ limit is derived in Ref. [29]. Its authors also show that in the heavy-quark limit, the current-current correlator can be analytically continued to Euclidean time, leading to the Euclidean color-electric correlator

$$
G_{\mathrm{E}}^{\mathrm{HQ}}(\tau)=-\sum_{i=1}^{3-2 \epsilon} \frac{\left\langle\operatorname{Re} \operatorname{Tr}\left[U(\beta, \tau) g_{\mathrm{B}} E_{i}(\tau, \mathbf{0}) U(\tau, 0) g_{\mathrm{B}} E_{i}(0, \mathbf{0})\right]\right\rangle}{3\langle\operatorname{Re} \operatorname{Tr}[U(\beta, 0)]\rangle} .
$$

For the details of the continuation, we refer the reader to the original paper [29].

In order to analytically continue $\gamma$, we need to relate the imaginary part of Eq. (3) with its analytic continuation Eq. (4). Therefore, we remind the reader of the relation between the imaginary part of a two-point function of two Hermitian operators $A, B$ in real time with the zero Matsubara frequency limit of the corresponding Euclidean correlator, such that

$$
\begin{aligned}
\operatorname{Im}_{R}^{A B}(\omega=0) & =\operatorname{Im} \int_{0}^{\infty} d t G^{A B}(t) \\
& =\int_{0}^{\beta} d \tau G_{E}^{A B}(\tau)=\tilde{G}_{E}\left(\omega_{n}=0\right) .
\end{aligned}
$$

The two-point functions are defined in the usual way, $G^{A B}(t)=i \operatorname{Tr}\{\hat{\rho}[A(t), B(0)]\}$ and $G_{E}^{A B}(\tau)=\operatorname{Tr}\{\hat{\rho} A(-i \tau) B(0)\}$, with $\hat{\rho} \equiv \frac{1}{Z} e^{-\beta H}$ the finite-temperature equilibrium density matrix. [Note that $\int_{0}^{\infty} d t G^{A B}(t)$ is purely imaginary since the commutator of Hermitian operators gives twice the imaginary part. Nevertheless, we take the imaginary part explicitly because the finite-frequency transform contains real and imaginary parts.]

We insert two complete sets of energy eigenstates in the definition of the two-point function such that the lhs of Eq. (5) becomes

$$
\begin{aligned}
& \int_{0}^{\infty} d t \sum_{n, m} \frac{2 i}{Z} \operatorname{Re}\left[A_{m n} B_{n m}\right] e^{-\frac{\beta}{2}\left(E_{n}+E_{m}\right)} \\
& \times \sinh \left(\frac{\beta\left(E_{n}-E_{m}\right)}{2}\right) e^{-i\left(E_{n}-E_{m}\right) t} \\
& \quad=\sum_{n, m} \frac{2}{Z} \operatorname{Re}\left[A_{m n} B_{n m}\right] \frac{e^{-\frac{\beta}{2}\left(E_{n}+E_{m}\right)}}{E_{n}-E_{m}} \sinh \left(\frac{\beta\left(E_{n}-E_{m}\right)}{2}\right),
\end{aligned}
$$

where we use the notation $A_{n m}=\langle n|A(t=0)| m\rangle$. Using the same procedure on the rhs of Eq. (5) yields

$$
\begin{aligned}
& \int_{0}^{\beta} d \tau \sum_{n, m} \frac{1}{Z} A_{m n} B_{n m} e^{-\beta E_{m}} e^{-\left(E_{n}-E_{m}\right) \tau} \\
& \quad=\sum_{n, m} \frac{2}{Z} A_{m n} B_{n m} \frac{e^{-\frac{\beta}{2}\left(E_{n}+E_{m}\right)}}{E_{n}-E_{m}} \sinh \left(\frac{\beta\left(E_{n}-E_{m}\right)}{2}\right) .
\end{aligned}
$$

So as long as $A_{m n} B_{n m}$ has no imaginary part, which happens if $A$ and $B$ are Hermitian operators or if $A=B^{\dagger}$, Eq. (5) is true. From this we conclude that the analytic continuation of the mass shift correlator $\gamma$ is

$$
\gamma=-\int_{0}^{\beta} d \tau G_{\mathrm{E}}^{\mathrm{HQ}}(\tau)
$$

where the minus sign emerges from different factors of $i$ in the definition of the color-electric field in real and imaginary time. The main result of this paper is therefore that the thermal effects on the mass shift $\gamma$ can be determined by a nonperturbative calculation using the vacuum subtracted Euclidean color-electric correlator on the lattice.

To further clarify the need for vacuum subtraction, let us look at the Euclidean color-electric correlator at leading order (LO), $\mathcal{O}\left(g^{2}\right)$. It is obtained trivially by connecting the two chromoelectric fields with a gluon propagator, yielding $[29,34]$

$$
G_{\mathrm{ELO}}^{\mathrm{HQ}}(\tau)=-\frac{g^{2} C_{F}}{3} y_{K} e^{i k_{n} \tau} \frac{(D-1) k_{n}^{2}+k^{2}}{k_{n}^{2}+k^{2}},
$$

where $\underset{K}{\mathbb{X}_{K}} \equiv T \sum_{k_{n}} \int_{k}, \int_{k} \equiv \int d^{d} k /(2 \pi)^{d}$ with $D=d+1$ the dimension of spacetime and $k_{n}$ the bosonic Matsubara frequency. One could immediately perform the $\tau$ integration of Eq. (6), obtaining $\beta \delta_{k_{n}}$, at which point the $\int_{k}$ integral would vanish in dimensional regularization (DR), as would, in this scheme, the vacuum contribution, where the $\tau$ integrations runs from $-\infty$ to $+\infty$ and the Matsubara sum is replaced with an integral over a continuous Euclidean frequency $k_{4}$. However, to better illustrate the need for vacuum subtraction in other schemes, such as the lattice, let us instead perform first the Matsubara sum and then the $\int_{k}$, which gives [29] 


$$
G_{\mathrm{ELO}}^{\mathrm{HQ}}(\tau)=g^{2} C_{F} \pi^{2} T^{4}\left[\frac{\cos ^{2}(\pi \tau T)}{\sin ^{4}(\pi \tau T)}+\frac{1}{3 \sin ^{2}(\pi \tau T)}\right] .
$$

The integration of this object over the compactified time direction does not converge, as the integrand diverges as $\tau^{-4}$ as $\tau \rightarrow 0$ and as $(\beta-\tau)^{-4}$ as $\tau \rightarrow \beta$. But this divergence is ultraviolet, as it comes about when the two $E$ fields are brought together. It is thus equal to the behavior observed in vacuum, which can be easily obtained from the $k_{4}$ integration, leading to

$$
G_{\mathrm{ELO}}^{\mathrm{HQ}}(\tau, T=0)=\frac{g^{2} C_{F}}{\pi^{2} \tau^{4}}
$$

Hence, vacuum subtraction in a non-DR scheme takes the form

$$
\begin{aligned}
\gamma= & -2 \int_{0}^{\beta / 2} d \tau\left[G_{\mathrm{ELO}}^{\mathrm{HQ}}(\tau)-G_{\mathrm{ELO}}^{\mathrm{HQ}}(\tau, T=0)\right] \\
& +2 \int_{\beta / 2}^{\infty} d \tau G_{\mathrm{ELO}}^{\mathrm{HQ}}(\tau, T=0)=0+\mathcal{O}\left(g^{4}\right),
\end{aligned}
$$

where we have exploited the symmetry of the thermal contribution at $\tau=\beta / 2$ and that of the vacuum at $\tau=0$. It is precisely a subtraction of this kind that would need to be performed on the lattice: for all $\tau<\beta / 2$ values, one computes the difference between the correlator on the thermal lattice and the vacuum lattice, and one then subtracts the integral of the vacuum contribution over $\tau>\beta / 2$. In practice, due to the noisy denominator in Eq. (4), it may be impossible to subtract $\gamma$ at very low temperatures on the lattice; in practice, a subtraction at a temperature where thermal effects are expected to be small should be sufficient.

At small separation, where the vacuum and thermal correlators diverge but the difference stays finite, it may be difficult to extract the difference with good statistical power. However, we believe that, while the individual short-distance values are sensitive to even small amounts of gradient flow [35-37], the difference should not be. This is supported by existing analytical studies [38], and it would be useful to investigate this issue further. We also refer to [39] for the perturbative $\mathcal{O}\left(\alpha_{\mathrm{s}}\right)$ renormalization of Eq. (4) in the lattice scheme.

\section{NLO INTEGRATION OF EUCLIDEAN CORRELATOR}

In this section, we validate our result in a perturbative calculation at next-to-leading order and present the imaginary-time integration of the correlator in Eq. (4). The Euclidean color-electric correlator was calculated in perturbation theory up to next-to-leading order in Ref. [34], and the contributing diagrams are shown in Fig. 1. Since we

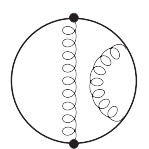

(b)

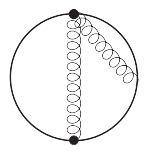

(g)

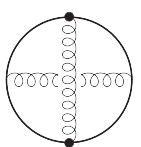

(c)

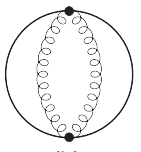

(h)

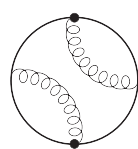

(d)

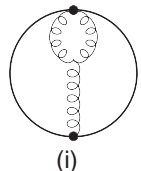

(i)

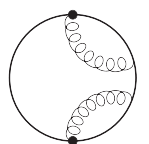

(e)
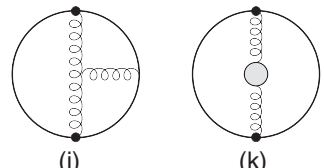

(k)
FIG. 1. Feynman diagrams contributing to the correlation function at order $g^{4}$. The circle represents the Polyakov line, and the heavy dots are the electric-field insertions. Diagram (k) includes the full gluon self-energy and therefore fermionic and ghost contributions. The labeling of the diagrams parallels that of Ref. [34]; diagram (a), not shown, is the order- $g^{2}$ graph.

are interested in the thermal contributions to $\gamma$, we subtract the vacuum contribution of the correlator during the calculation. As previously highlighted, dimensional regularization does that automatically, so in the following we will not keep track of scale-free contributions that vanish in any $D$.

Using the integral expression of $G_{\mathrm{ENLO}}^{\mathrm{HQ}}(\tau)$ obtained in Ref. [34] from the diagrams above, we notice that the only $\tau$ dependence is in the Fourier transform. After applying the Kronecker delta $\beta \delta_{k_{n}}$ arising from the $\tau$ integration, we obtain that

$$
\begin{aligned}
\gamma_{\mathrm{LO}} & =-\int_{0}^{\beta} d \tau G_{\mathrm{ENLO}}^{\mathrm{HQ}}(\tau) \\
& =\frac{g^{4} C_{F}}{3}\left[N_{c}\left(\mathcal{I}_{1}+2(D-2) \mathcal{I}_{2}\right)-N_{f}\left(\tilde{\mathcal{I}}_{1}+4 \tilde{\mathcal{I}}_{2}\right)\right],
\end{aligned}
$$

where we have introduced the sum integrals

$$
\begin{aligned}
& \mathcal{I}_{1}, \tilde{\mathcal{I}}_{1}=\left.\int_{k} \sum_{Q,\{Q\}} \frac{1}{Q^{2}(K-Q)^{2}}\right|_{k_{n}=0},
\end{aligned}
$$

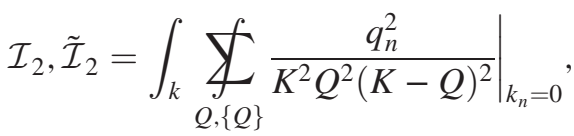

where $K^{2}=k_{n}^{2}+\mathbf{k}^{2}$. The notation $\{Q\}$ represents the fermionic Matsubara frequencies $q_{n}=\pi T(2 n+1)$ for the fermionic sum integrals, denoted by $\tilde{\mathcal{I}}$. These are directly related to the bosonic ones via

$$
\sigma_{f}(T)=T \sum_{\left\{q_{n}\right\}} f\left(q_{n}, \ldots\right)=2 \sigma_{b}(T / 2)-\sigma_{b}(T) .
$$


The bosonic integrals are easily evaluated as

$$
\begin{gathered}
\mathcal{I}_{1}=\frac{\Gamma^{2}(1-d / 2) \zeta(4-2 d)}{8 \pi^{4-d} T^{3-2 d}} \stackrel{\mathrm{d} \rightarrow 3}{=} 0, \\
\mathcal{I}_{2}=-\frac{(d-2) \Gamma^{2}(1-d / 2) \zeta(4-2 d)}{16(d-3) \pi^{4-d} T^{3-2 d}} \stackrel{\mathrm{d} \rightarrow 3}{=}-\frac{\zeta(3)}{8 \pi^{2} \beta^{3}},
\end{gathered}
$$

where we have used a Feynman parameter for $\mathcal{I}_{2}$. The fermionic counterparts, obtained from Eq. (14), are

$$
\mathcal{I}_{a} \propto \frac{1}{\beta^{2 d-3}} \rightarrow \tilde{\mathcal{I}}_{a}=\mathcal{I}_{a} \times\left(4^{2-d}-1\right)
$$

Putting everything together, we find

$$
\gamma_{\mathrm{LO}}=-2 \alpha_{\mathrm{s}}^{2} T^{3} \zeta(3) C_{F}\left(\frac{4}{3} N_{\mathrm{c}}+N_{\mathrm{f}}\right) .
$$

This is the known result from the real-time calculation of the mass shift in quarkonium given in Ref. [10]. Consequently, we have shown that the imaginary time correlator in Eq. (4) reproduces the leading-order result of Eq. (2).

Interestingly, it is also possible to check the agreement beyond LO. Our analysis has so far used unresummed perturbation theory, which is appropriate when all momenta are of order $T$ and the Matsubara frequency $q_{n}$ is nonzero. Unlike $\kappa$, which receives a contribution from the $g T$ scale at LO, $\gamma$ does not. Inspection of Eqs. (6) and (7) shows that $k \sim g T$ contributes to $\gamma$ at $\mathcal{O}\left(g^{5}\right)$. This contribution is easily obtained by replacing Eq. (7) with its resummed version. Since the $\tau$ integration forces $k_{n}=0$, it suffices to use electrostatic QCD (EQCD) [40-44], where $\mathbf{E} \approx-i \boldsymbol{\nabla} A_{0}$. Then the temporal component of the gauge field gets Debye-screened, yielding

$$
\gamma_{\mathrm{NLO}}=\frac{g^{2} C_{F}}{3} \int_{k} \frac{k^{2}}{k^{2}+m_{D}^{2}}=\frac{\alpha_{\mathrm{s}} C_{F} m_{D}^{3}}{3},
$$

where $m_{D}^{2}=g^{2} T^{2}\left(N_{\mathrm{c}} / 3+N_{\mathrm{f}} / 6\right)$ is the Debye mass. Equation (18) agrees with Eq. (87) of [10], recalling that $\operatorname{Re} \delta V_{s}(r)_{11}^{[10]}=\gamma r^{2} / 2$. This $\mathcal{O}\left(g^{5}\right)$ term is the only NLO contribution to $\gamma$; to the best of our knowledge, this was not observed in the previous literature.

\section{DISCUSSION AND CONCLUSIONS}

In this paper, we showed that the coefficient $\gamma$, introduced by Brambilla, Escobedo, Soto, and Vairo [14], and representing a thermal medium effect on the heavy quark bound state energies, can be reexpressed in terms of a Euclidean correlation function, (4), which is highly amenable to a lattice determination. With the vacuum contributions removed, the time integral of the correlator, (6), should not suffer from divergences, and the computational cost should be reasonable if smoothing techniques like gradient flow are employed. We confirmed that the LO results for $\gamma$, evaluated via real-time techniques in Ref. [10], agree with the Euclidean time integration of the results of Ref. [34], which is a nontrivial check on our derivation. We also obtained the NLO correction to $\gamma$ in Eq. (18).

\section{ACKNOWLEDGMENTS}

We thank the Technische Universität Darmstadt and its Institut für Kernphysik, where this work was conducted and where J. G. was hosted during the early phase of this work. This work was funded by the Deutsche Forschungsgemeinschaft (DFG, German Research Foundation) Project No. 315477589 TRR 211.

Note added.-Recently, we became aware of the preprint "Transport coefficients from in medium quarkonium dynamics" [45] by N. Brambilla, M. Á. Escobedo, A. Vairo, and P. Vander Griend. It proposes a way to determine $\gamma$ from the quarkonium spectral function reconstructed from lattice QCD [46]. We thank the authors for sharing their results with us prior to publication and for discussion.
[1] A. Andronic et al., Eur. Phys. J. C 76, 107 (2016).

[2] T. Matsui and H. Satz, Phys. Lett. B 178, 416 (1986).

[3] A. Adare et al. (PHENIX Collaboration), Phys. Rev. C 91, 024913 (2015).

[4] L. Adamczyk et al. (STAR Collaboration), Phys. Rev. C 90, 024906 (2014).

[5] L. Adamczyk et al. (STAR Collaboration), Phys. Lett. B 735, 127 (2014); 743, 537(E) (2015).

[6] B. B. Abelev et al. (ALICE Collaboration), Phys. Lett. B 734, 314 (2014).
[7] J. Adam et al. (ALICE Collaboration), Phys. Lett. B 766, 212 (2017).

[8] V. Khachatryan et al. (CMS Collaboration), Phys. Lett. B 770, 357 (2017).

[9] R. Rapp and X. Du, Nucl. Phys. A967, 216 (2017)..

[10] N. Brambilla, J. Ghiglieri, A. Vairo, and P. Petreczky, Phys. Rev. D 78, 014017 (2008).

[11] N. Brambilla et al., Eur. Phys. J. C 71, 1534 (2011).

[12] A. Mocsy, P. Petreczky, and M. Strickland, Int. J. Mod. Phys. A 28, 1340012 (2013). 
[13] Y. Burnier, O. Kaczmarek, and A. Rothkopf, J. High Energy Phys. 12 (2015) 101.

[14] N. Brambilla, M. A. Escobedo, J. Soto, and A. Vairo, Phys. Rev. D 96, 034021 (2017).

[15] N. Brambilla, M. A. Escobedo, J. Soto, and A. Vairo, Phys. Rev. D 97, 074009 (2018).

[16] C. Young and E. Shuryak, Phys. Rev. C 81, 034905 (2010).

[17] X. Zhao and R. Rapp, Nucl. Phys. A859, 114 (2011).

[18] J.-P. Blaizot, D. De Boni, P. Faccioli, and G. Garberoglio, Nucl. Phys. A946, 49 (2016).

[19] A. Pineda and J. Soto, Nucl. Phys. B, Proc. Suppl. 64, 428 (1998).

[20] N. Brambilla, A. Pineda, J. Soto, and A. Vairo, Nucl. Phys. B566, 275 (2000).

[21] N. Brambilla, A. Pineda, J. Soto, and A. Vairo, Rev. Mod. Phys. 77, 1423 (2005).

[22] J. Casalderrey-Solana and D. Teaney, Phys. Rev. D 74, 085012 (2006).

[23] B. Svetitsky, Phys. Rev. D 37, 2484 (1988).

[24] G. D. Moore and D. Teaney, Phys. Rev. C 71, 064904 (2005).

[25] S. Caron-Huot and G. D. Moore, Phys. Rev. Lett. 100, 052301 (2008).

[26] S. Caron-Huot and G. D. Moore, J. High Energy Phys. 02 (2008) 081.

[27] H. van Hees, M. Mannarelli, V. Greco, and R. Rapp, Phys. Rev. Lett. 100, 192301 (2008).

[28] C. P. Herzog, A. Karch, P. Kovtun, C. Kozcaz, and L. G. Yaffe, J. High Energy Phys. 07 (2006) 013.

[29] S. Caron-Huot, M. Laine, and G. D. Moore, J. High Energy Phys. 04 (2009) 053.
[30] H. T. Ding, A. Francis, O. Kaczmarek, F. Karsch, H. Satz, and W. Soldner, J. Phys. G 38, 124070 (2011).

[31] A. Francis, O. Kaczmarek, M. Laine, and J. Langelage, Proc. Sci., LATTICE2011 (2011) 202.

[32] D. Banerjee, S. Datta, R. Gavai, and P. Majumdar, Phys. Rev. D 85, 014510 (2012).

[33] A. Francis, O. Kaczmarek, M. Laine, T. Neuhaus, and H. Ohno, Phys. Rev. D 92, 116003 (2015).

[34] Y. Burnier, M. Laine, J. Langelage, and L. Mether, J. High Energy Phys. 08 (2010) 094.

[35] R. Narayanan and H. Neuberger, J. High Energy Phys. 03 (2006) 064.

[36] M. Lüscher, Commun. Math. Phys. 293, 899 (2010).

[37] M. Lüscher, J. High Energy Phys. 08 (2010) 071; 03 (2014) 92.

[38] A. M. Eller and G. D. Moore, Phys. Rev. D 97, 114507 (2018).

[39] C. Christensen and M. Laine, Phys. Lett. B 755, 316 (2016).

[40] E. Braaten, Phys. Rev. Lett. 74, 2164 (1995).

[41] E. Braaten and A. Nieto, Phys. Rev. D 51, 6990 (1995).

[42] E. Braaten and A. Nieto, Phys. Rev. D 53, 3421 (1996).

[43] K. Kajantie, M. Laine, K. Rummukainen, and M. E. Shaposhnikov, Nucl. Phys. B458, 90 (1996).

[44] K. Kajantie, M. Laine, K. Rummukainen, and M. E. Shaposhnikov, Nucl. Phys. B503, 357 (1997).

[45] N. Brambilla, M. A. Escobedo, A. Vairo, and P. Vander Griend, Technical University of Munich Report No. TUMEFT 122/18, 2019.

[46] S. Kim, P. Petreczky, and A. Rothkopf, J. High Energy Phys. 11 (2018) 088. 